\title{
Investigation on the Inhibitive Properties of Some Selected Agro Food Extracts on Oil and Gas Pipeline
}

\author{
M.A. Bodude ${ }^{1 *}$, S.O. Oyegbami ${ }^{2}$ and O.R. Oloyede ${ }^{3}$ \\ 1. Department of Metallurgical and Materials Engineering, University of Lagos, Lagos, Nigeria \\ 2. Department of metallurgical Engineering, Yaba College of Technology, Yaba. Lagos, Nigeria. \\ 3. School of Maritime Engineering Academy, Oron. Akwa Ibom, Nigeria
}

\begin{abstract}
In this research work, three agro food (cocoa, kola and bitter kola) extract were tested for their corrosion inhibition efficiency on a mild steel pipeline material in $0.05 \mathrm{M} \mathrm{HCl}$ acid using weight loss measurement technique. The results obtained showed that the Cocoa extract gives the highest inhibition efficiency of $85 \%$ at the concentration of $0.4 \mathrm{~mL} / \mathrm{L}$.
\end{abstract}

Key words: Corrosion, agro-food extract, inhibitor, pitting.

\section{Introduction}

Corrosion has been identified as one of the major problems in the oil and gas industry, especially in the transportation pipeline. Several millions of dollars is reported to be spent annually in combating this problem world-wide.

Carbon steel is being commonly used for producing oil and gas transportation pipelines. Internal corrosion has been encountered with carbon steel for many years in oil and gas production [1]. Internal pipeline corrosion is primarily associated with the presence of free water, which is potentially corrosive with the existence of corrosive species, like $\mathrm{CO}_{2}, \mathrm{HS}_{2}$, organic acids, etc..

Corrosion control and prevention are major scientific issues that must be addressed daily as far as there are increasing needs of metallic materials in all facets of technological development. In the oil and gas industries, mild steels are found useful in many applications, such as in pipeline, vessels, storage tanks, well-heads, and so on. It is also extensively used in the form of flat and rolled plate in the construction of offshore production platforms, steel jackets and

Corresponding author: M.A. Bodude. E-mail: mbodude@unilag.edu.ng. drilling rigs along with piping systems that are subjected to rigorous grit blasting followed by anticorrosion coatings. This is to combat the decks being sprayed by sea water and salt laden air produced by gale force winds.

The use of inhibitors for the control of corrosion of metals and alloys which are in contact with aggressive environment is one among the acceptable practices used to reduce and/or prevent corrosion.

The corrosion inhibition of steels has received a considerable amount of attention as a result of its industrial concern [2, 3]. Extracts of some common plants and plant products have been tried as corrosion inhibitors for metals and alloys under different environment [4]. Alkaloids extract from Annona squamosa plant have been studied as possible corrosion inhibitor for C38 steel in molar hydrochloric acid (1 M $\mathrm{HCl})$. The corrosion inhibition efficiency was found to increases on increasing plant extract concentration. The inhibition efficiency of Annona squamosa extract was temperature dependent and its addition led to an increase in the activation corrosion energy revealing a physical adsorption between the extract and the metal surface. The inhibition efficiency obtained from impedance and polarization measurements was in good agreement and was found to increase with increasing 
concentration of alkaloids extract up to $50 \mathrm{mg} / \mathrm{L}$ for stems extract. The adsorption of the extract on the low carbon steel surface obeys the Langmuir adsorption [5].

The inhibitive action of eggplant peel extract toward the corrosion of mild steel in $2 \mathrm{~mol} \mathrm{HCl}$ solution using weight loss measurements and electrochemical techniques was carried out. The results of both methods showed that the corrosion rate is decreasing and inhibition efficiency is increasing as the concentration of the eggplant inhibitor increased. The adsorption data were analyzed using various adsorption isotherm models and the results at temperatures of $25^{\circ} \mathrm{C}, 40^{\circ} \mathrm{C}$ and $50{ }^{\circ} \mathrm{C}$ have shown that the adsorption behaviour of eggplant extract molecules is best described by the Langmuir adsorption isotherm [6]. Gravimetric method was used to study the temperature effects on mild steel corrosion in $2.0 \mathrm{~mol}$ of $\mathrm{HCl}$ and $\mathrm{H}_{2} \mathrm{SO}_{4}$ in the absence and presence of aqueous extract for fenugreek leaves (AEFL). In $2.0 \mathrm{~mol} \mathrm{HCl}$, the results revealed that the inhibition efficiency of AEFL increases with increasing concentration, but an increase or decrease in the inhibitor efficiency depending on its concentration is detected with increasing temperature. On the other hand, in $2.0 \mathrm{~mol} \mathrm{H}_{2} \mathrm{SO}_{4}$, the inhibition efficiency of AEFL was found to increase with increasing both the inhibitor concentration and solution temperature. The inhibition mechanism for the adsorption of AEFL species on mild steel surface from both acids was discussed with the light of some AEFL constituents [7].

The corrosion inhibition performance in almost all the cited plant extracts could be due to the presence of surface active constituents which normally enhance the film formation over the metal surface, thus mitigating corrosion. Inspection of the chemical structures of some of the constituents of the plants extracts reveal that all molecules are long chain hydrocarbons carrying a polar group(s) at one or eitherends. The polar groups normally contain oxygen, nitrogen or sulphur atoms. Among many methods of corrosion control and prevention the organic inhibitors are the most frequently used [8]. Organic compounds used as inhibitors act through a process of surface adsorption, so the efficiency of an inhibitor depends on [9]:

(1) the chemical structure of the organic compound;

(2) the surface charge of the metal;

(3) the type of interactions between the organic molecule and metal surface.

Existing data reveal most inhibitors to act by adsorption on the metal surface through heteroatoms such as nitrogen, oxygen and sulphur, double bonds, triple bonds or aromatic rings which tend to form stronger coordination bonds.

This research work is aimed at using the extract of kola juice, bitter kola juice and cocoa juice as inhibitors against corrosion of oil and gas transportation pipelines with a view to determining the inhibition efficiencies of the extracts.

\section{Materials and Methods}

The pipeline steel specimen used for this work has a nominal percentage composition of: $0.15 \mathrm{C}, 0.20 \mathrm{Si}$, 0.04 S, $0.85 \mathrm{Mn}, 0.10 \mathrm{Ni}, 0.20 \mathrm{Cr}, 0.02 \mathrm{Mo}, 0.001 \mathrm{~V}$, $0.35 \mathrm{Cu}$, the rest being $\mathrm{Fe}$. The Corrosion inhibition efficiency of fruits extracts was evaluated using weight loss measurements for effect of various solution concentrations. The steel pipe was cut into pieces of different lengths and prepared for corrosion (weight loss) test. The test was performed by dipping the steel samples in 0.05M HCL with the extracted agro food solutions (inhibitors) added in different concentrations. The specimens were removed from the solution every 3 days, washed with distilled water, rinsed with ethanol, air-dried, and re-weighed. Plots of weight loss versus the exposure time and of calculated corrosion rate versus time of exposure were made. All the experiments were performed at ambient temperature(s).

Corrosion rate (CR) and inhibition efficiencies (I.E, \%) were derived from Eq. (1) and Eq. (2), respectively.

$$
\mathrm{CR}=\mathrm{W}_{\mathrm{o}}-\mathrm{W}_{\mathrm{i}} / \mathrm{At}
$$

where $\mathrm{W}_{\mathrm{o}}$ and $\mathrm{W}_{\mathrm{i}}$ are respectively the weights of the 
specimen before and after exposure to $0.5 \mathrm{M} \mathrm{Hcl}$, A, is the total surface area, and $t$, is the time of exposure in hours.

$$
\mathrm{IE} \%=\mathrm{CR}_{0}-\mathrm{CRi} / \mathrm{CR}_{0} \times 100
$$

where $\mathrm{CR}_{0}$ and $\mathrm{CR}_{\mathrm{i}}$ are the corrosion rates of mild steel in $0.5 \mathrm{M} \mathrm{HCl}$ without and in the presence of various concentrations of different inhibitors, respectively.

\section{Results and Discussion}

The results of the corrosion experiment are shown in the figures above. Figs. 1-3 show the Corrosion rates of the pipeline steel exposed to $0.05 \mathrm{M} \mathrm{HCl}$ with various concentrations of the agro food extracts used as inhibitors for 3, 6 and 9 days, respectively. From all the plots, corrosion rates reduce with increasing inhibitors concentrations for both cocoa and kola extracts, while that of the bitter kola increases. Generally the corrosion rates are minimal in the acid solution with the cocoa extract and least rate occurred at about $80 \%-85 \%$ concentration of the extract. These behaviours indicate that cocoa seed extract is a very potent corrosion inhibitor followed by kola fruit extract especially when used at about $80 \%-85 \%$ concentration.

Figs. 4-6 reveal the corrosion inhibition efficiencies of the three agro extracts on the mild steel in acid media at different concentrations and for 3, 6 and 9 days exposure respectively. Again the inhibition efficiencies of cocoa extract was found to be about $85 \%$ and this is the highest of all the three extracts followed by kola extract which is about $30 \%$ while bitter kola extract is about negative $50 \%$ at 3 days of exposure and with about $80 \%-85 \%$ of the inhibitors in

Table 2 Chemical analysis of cocoa extract.

\begin{tabular}{lll}
\hline Elements & Theobromide & Polyphenols \\
\hline Composition (\%) & 32 & 12.1 \\
\hline
\end{tabular}

Table 3 Chemical analysis of kola extract.

\begin{tabular}{llll}
\hline Elements & Caffeine & Theobromine & Tannin \\
\hline Composition (\%) & $2.0-3.5$ & $1.0-2.5$ & 0.01 \\
\hline
\end{tabular}

Table 4 Chemical analysis of Bitter kola extract.

\begin{tabular}{lll}
\hline Elements & Theobromine & Tannin \\
\hline Composition (\%) & 3.61 & 0.092 \\
\hline
\end{tabular}

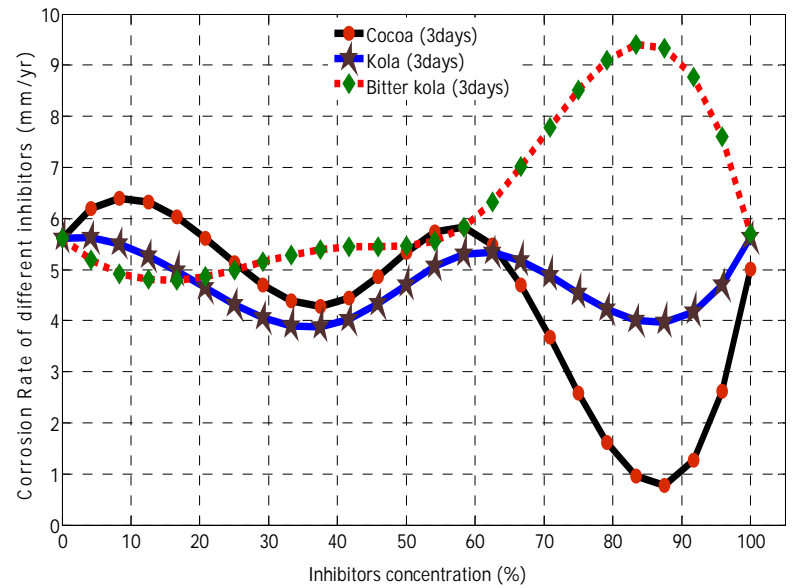

Fig. 1 Corrosion rates of mild steel exposed to various locally made inhibitors with varied concentration for three days.

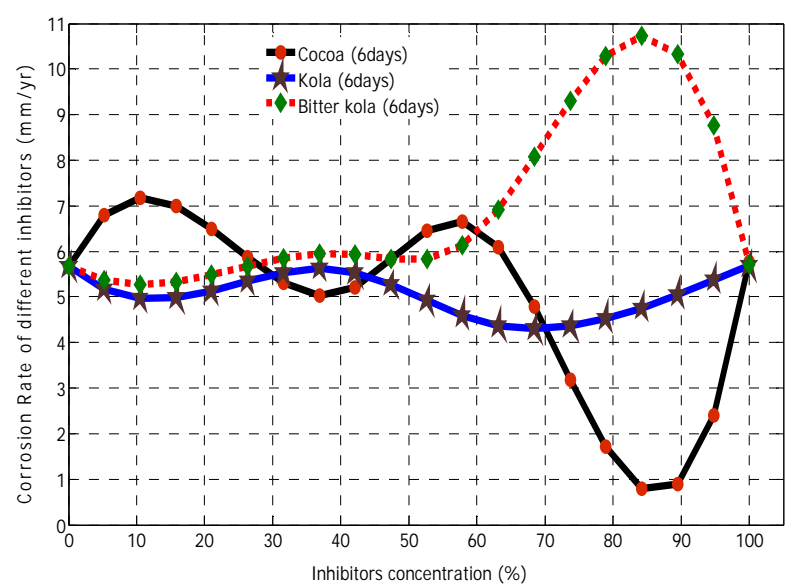

Fig. 2 Corrosion rates of mild steel exposed to various locally made inhibitors with varied concentration for six days.

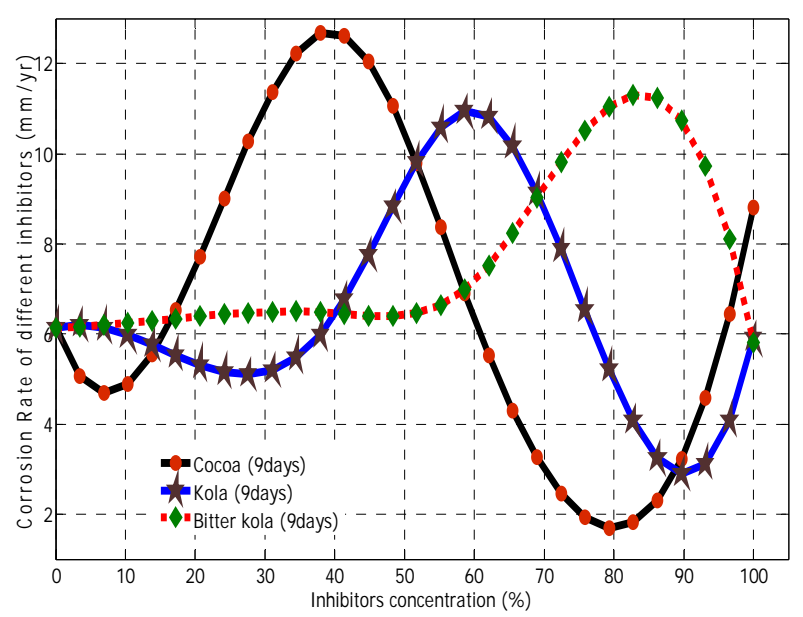

Fig. 3 Corrosion rates of mild steel exposed to various locally made inhibitors with varied concentration for nine days. 


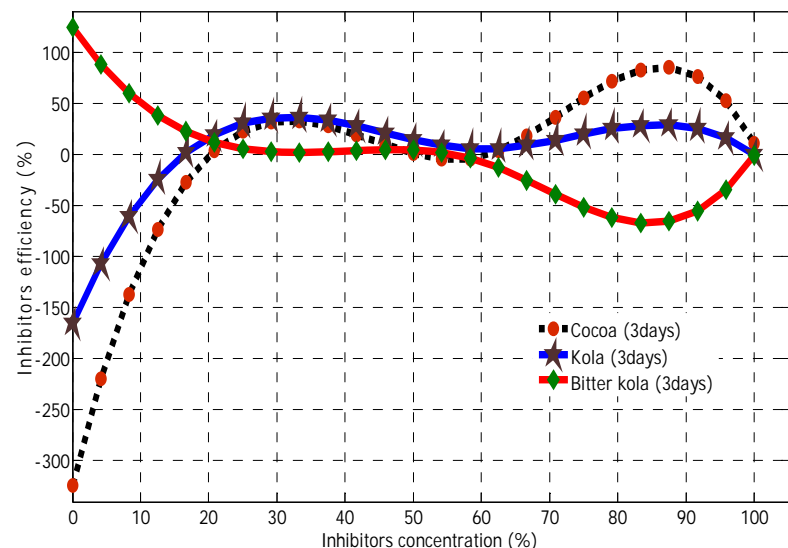

Fig. 4 Inhibitor efficiency of the extracts exhibited for three days.

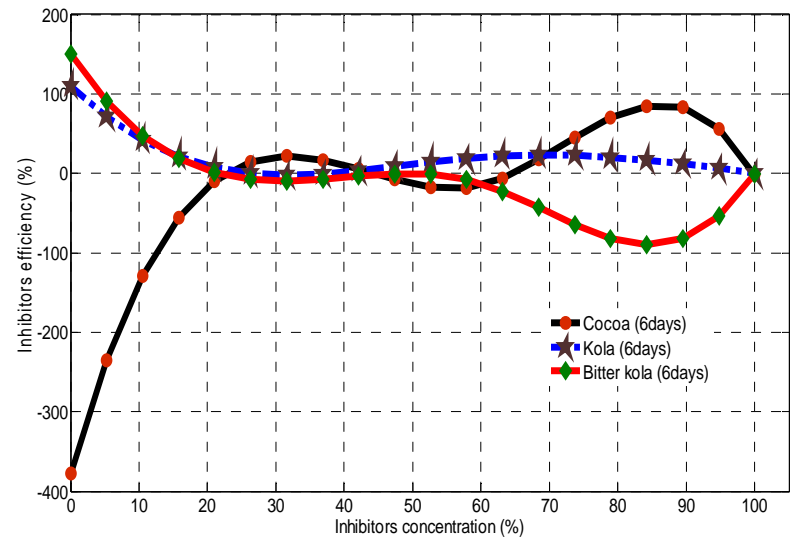

Fig. 5 Inhibitor efficiency of the extracts exhibited for six days.

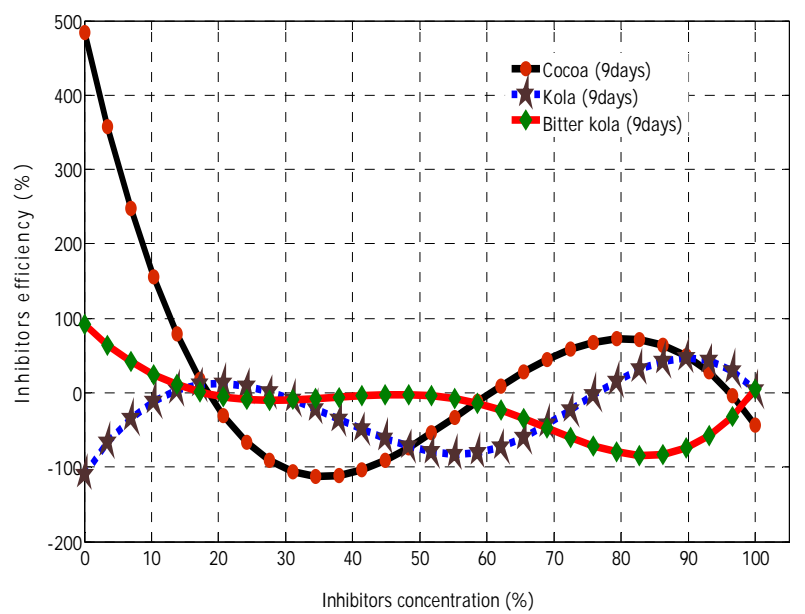

Fig. 6 Inhibitor efficiency of the extracts exhibited for nine days.

the acid media. This finding is significant in confirming the potency of cocoa fruit extract as corrosion inhibitor while the corrosiveness of bitter kola is also revealed.

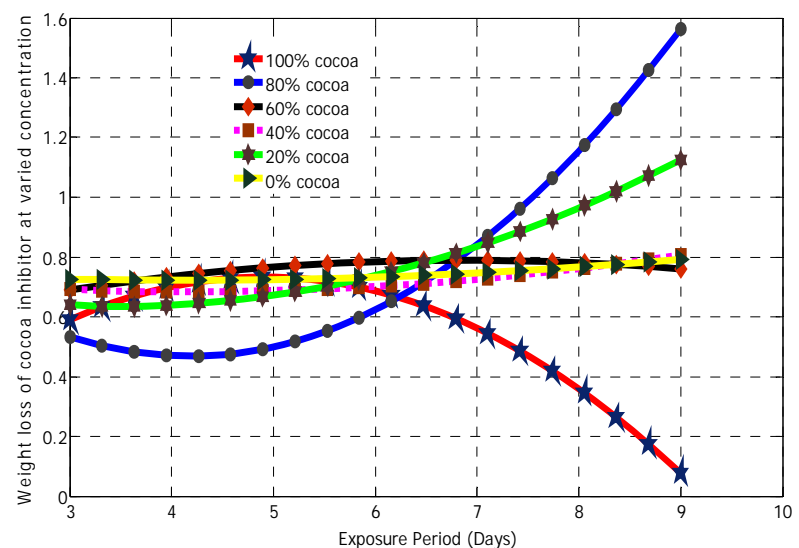

Fig. 7 Weight loss of mild steel at different concentrations of cocoa extract exhibited for nine days

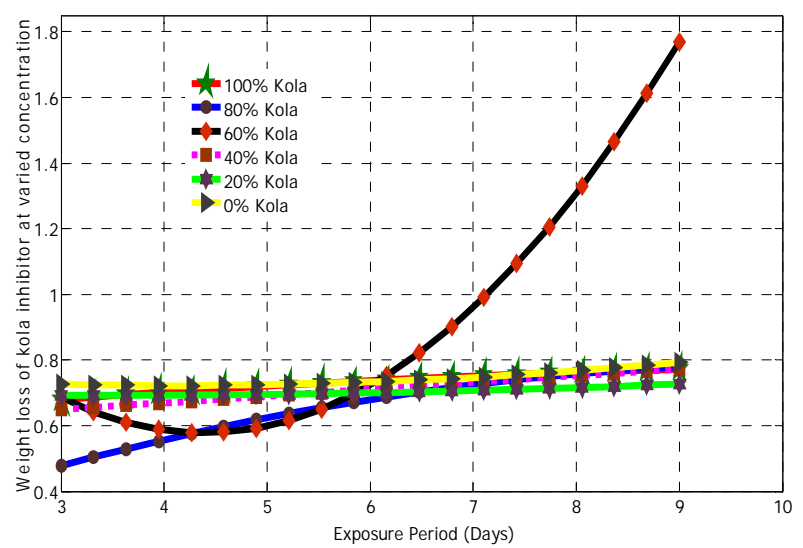

Fig. 8 Weight loss of mild steel at different concentrations of kola extract exhibited for nine days.

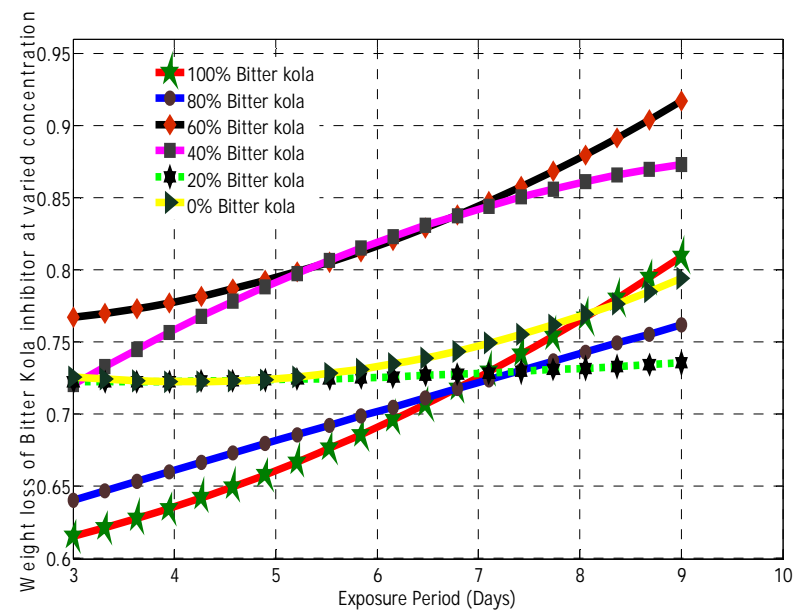

Fig. 9 Weight loss of mild steel at different concentration of bitter kola extract exhibited for nine days

Figs. 7-9 are plots of weight loss data against exposure time obtained from the experiment from which the above corrosion rates were computed. 


\section{Conclusions}

This work has been able to show that corrosion of oil and gas facilities especially the transportation pipelines and storage tanks can be effectively minimised using cocoa fruit extract which has about $85 \%$ inhibition efficiency when used within 3 to 6 days and at about $80 \%$ concentration. Also the work showed the negative corrosion effect of bitter kola extract on the pipeline material .Hence cocoa fruit extract is recommended as inhibitor for mitigating against corrosion of oil and gas facilities. However, further work should be carried out to study the chemical compatibilities and stability of this extract with various oil and gas products to avoid contamination.

\section{References}

[1] Smith, L. 1999. "Control of Corrosion in Oil and Gas Production Tubing.” British Corrosion Journal 34:
247-253.

[2] González-Rodriguez, E., Uruchurtu, J. G., Sarmiento, J., and Menchaca, O. 2009. MInt. J. Electrochem. Sci. 4: 134.

[3] Freirea, L., Nóvoaa, X. R., Montemorb, M. F., and Carmezimb, M. J. 2009. Mat. Chem. and Phy. 114: 962.

[4] Singh, A., Ebenso, E. E., and Quraishi, M. A. 2011. "Corrosion Inhibition of Carbon Steel in HCl Solution by Some Plant Extracts."

[5] Lebrini1, Robert2, M. F., and Roos2, C. 2010. "Inhibition Effect of Alkaloids Extract from Annona Squamosa Plant on the Corrosion of c38 STeel in Normal Hydrochloric Acid Medium.".

[6] Taleb, I., and Mehad, H. 2011. "Corrosion Inhibition of Mild STEel in 2m Hcl Using Aqueous Extract of Eggplant Peel.”

[7] Ehteram, A. N. 2011. "Temperature Effects on the Corrosion Inhibition of Mild Steel in Acidic Solutions by Aqueous Extract of Fenugreek Leaves.” 20 October 2011

[8] Haleem, Abd El Wanees, S., Abd El Aal, E. E., and Diab, A. 2010. Corrosion Sci. 52: 292.

[9] Ergun, Ü., Yüzer, D., Emregül, K. C. 2008. Mat. Chem. Phy. 109: 492. 